\title{
Leveraging immunochemotherapy for treating pancreatic
} cancer

\author{
Anushka Dongre ${ }^{1}$ and Robert A. Weinberg ${ }^{1,2,3}$ 瓜 \\ (C) CEMCS, CAS 2021
}

Cell Research (2021) 31:1228-1229; https://doi.org/10.1038/s41422-021-00574-x

\begin{abstract}
The refractory response of pancreatic ductal adenocarcinoma (PDAC) to multiple treatment regimens can be attributed to the presence of a desmoplastic stroma together with an immunosuppressive tumor microenvironment. In a recent study published in Cell, Koikawa et al. demonstrate that targeting the proline isomerase, Pin 1, potentiates the efficacy of immunochemotherapy and enhances the susceptibility of highly resistant PDAC to the anti-tumor immune response.
\end{abstract}

Harnessing the immune response as a strategy to eliminate certain cancers holds the promise of revolutionizing cancer treatment. T cells express several receptors such as PD1 and CTLA4 that act to suppress T cell function upon engagement with their cognate ligands, many of which are expressed by cancer cells themselves. Inhibition of these juxtacrine signaling interactions by the use of anti-PD1 or anti-CTLA4 "checkpoint" antibodies can restore $T$ cell function and subsequently their ability to eliminate carcinomas. Checkpoint blockade immunotherapies administered individually or in combination have been successful in treating a small proportion of cancers, specifically, certain lung cancers and melanoma. ${ }^{1}$ However, the responses of some other cancer types, such as those of the pancreas, are limited. ${ }^{2}$

The refractory responses of pancreatic ductal adenocarcinoma (PDAC) to immunotherapy, chemotherapy, and targeted therapies can be attributed to the presence of (i) a highly desmoplastic stroma comprised of cancer-associated fibroblasts (CAFs); and (ii) an immunosuppressive tumor microenvironment (TME) comprised of myeloid-derived suppressor cells (MDSCs), alternatively activated M2-like macrophages, and regulatory T cells (Tregs). The presence of these cells represents an important element in tumor pathogenesis, because they secrete multiple immunosuppressive cytokines and can thereby attenuate the actions of cytotoxic $\mathrm{CD}^{+}$ $T$ cells and NK cells, which might otherwise be capable of mounting effective anti-tumor immune responses.

Previous attempts at targeting stromal CAFs have generated conflicting results, with some strategies leading to tumor regression and yet others resulting in PDAC acceleration. ${ }^{3,4}$ These limitations bring to the forefront the need to identify additional strategies that can disrupt the desmoplastic and immunosuppressive TME of PDAC and potentiate the efficacy of currently administered treatment regimens. In a recent study published in Cell, Koikawa et al. demonstrate that targeting the proline isomerase, Pin1, generates synergistic responses with both antiPD1 and gemcitabine in multiple models of PDAC. ${ }^{5}$ These responses, as the authors demonstrate, can be ascribed to the actions of Pin 1 inhibitors on the CAFs forming the tumorassociated stroma as well as the cancer cells themselves (Fig. 1).

To begin, the authors reasoned that while Pin1 was known to promote tumor progression by regulating the expression of multiple oncogenic signaling pathways, ${ }^{6,7}$ its possible actions in regulating the assembly of a desmoplastic and immunosuppressive TME were unexplored. For this reason, patient-derived organoids as well as an orthotopic allograft model of PDAC in syngeneic, immunocompetent hosts were treated with two different pharmacologic inhibitors of Pin1. As the authors found, Pin 1 inhibition blocked CAF activation, reduced the numbers of immunosuppressive Tregs and myeloid cells, and resulted in increased numbers of cytotoxic $\mathrm{CD}^{+} \mathrm{T}$ cells compared to untreated controls. To determine whether these aforementioned changes in the TME enhanced the susceptibility of PDAC to immunochemotherapy, the authors treated PDAC tumor-bearing mice with Pin1 inhibitors (Pin1i) in combination with anti-PD1 and/or gemcitabine. Strikingly, $87.5 \%$ of mice treated with the triple combination of Pin $1 \mathrm{i}+$ anti-PD1 + gemcitabine showed complete tumor regression and long-term, tumor-free survival relative to tumor-bearing mice that had received single or double agent therapy. Similar tumor regression was also observed in a genetically engineered mouse model of autochthonous PDAC that was administered triple agent therapy. Thus, targeting Pin1 dramatically enhanced the susceptibility of previously refractory PDAC to immunochemotherapy.

To determine the source of Pin 1 within the TME, the authors analyzed Pin1 expression in surgically resected human tumor tissues from 167 PDAC patients and found that Pin1 was expressed strongly by both cancer cells and stromal CAFs. Furthermore, high Pin1 expression correlated poorly with patient survival and was accompanied by the presence of immunosuppressive Tregs, M2-like macrophages and CAFs relative to Pin1-low tumors, which were infiltrated by $\mathrm{CD}^{+}$cytotoxic $\mathrm{T}$ cells instead. The authors undertook to determine whether the enhanced responsiveness to immunochemotherapy was driven primarily by inhibition of Pin1 in CAFs or, alternatively, whether such responsiveness stemmed entirely from inhibition of carcinoma cell-intrinsic Pin1. Abrogation of Pin 1 in CAFs impaired their ability to secrete multiple paracrine factors. Prominent amongst these was the immunosuppressive cytokine TGF $\beta$, which is known to promote an immunosuppressive TME. ${ }^{8}$ In addition, Pin 1 inhibition

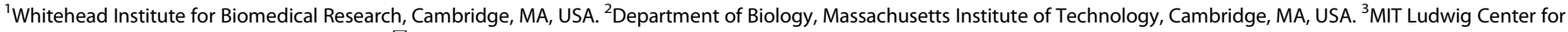
Molecular Oncology, Cambridge, MA, USA. ${ }^{\bowtie}$ email: weinberg@wi.mit.edu 
Pin1 expression in the TME

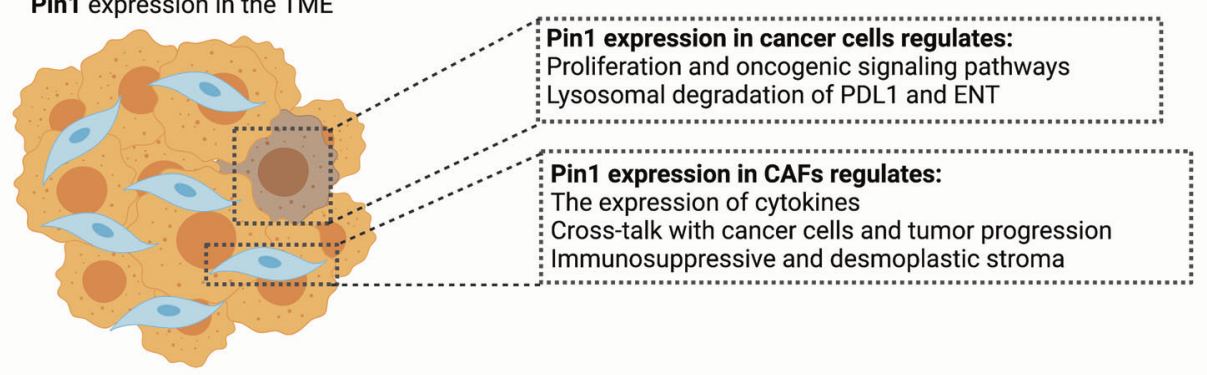

Unresponsive to

anti-PD1+Gemcitabine

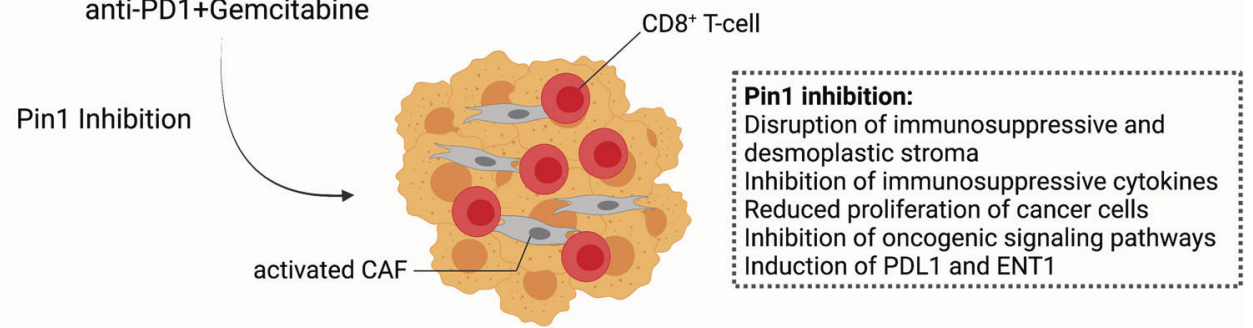

Responsive to

Created using BioRender anti-PD1+Gemcitabine

Fig. 1 The role of Pin 1 in the PDAC TME. Inhibition of Pin 1 potentiates the efficacy of immunochemotherapy and leads to tumor regression in multiple models of PDAC by disrupting the desmoplastic and immunosuppressive TME.

in CAFs also impaired the proliferation of cancer cells themselves. Thus, it is likely that CAF-derived Pin1 drives the expression of multiple paracrine factors that impinge on both immune cells and cancer cells within the TME.

In a parallel set of experiments, the authors also asked whether similar effects would be observed if Pin1 expression was abrogated in cancer cells instead of CAFs. Abrogation of Pin1 in cancer cells impaired their proliferation and simultaneously induced surface expression of ENT1 (a transporter that facilitates the uptake of gemcitabine) as well as PDL1 (the ligand for PD1, an inhibitory receptor expressed on the surface of $T$ cells). Thus, inhibition of Pin1 in carcinoma cells induced their upregulation of two proteins, ENT1 and PDL1, which likely renders them more susceptible to elimination by immunochemotherapy. The authors proceeded to examine the mechanism underlying Pin1-mediated expression of ENT1 and PDL1 and uncovered that Pin1 promotes endocytosis and lysosomal degradation of both proteins by acting on the pSer929-Pro motif in HIP1R, which is known to regulate lysosomal degradation of PDL1. ${ }^{9}$

In summary, this study provides insights into the heterotypic signaling interactions that operate within the TME of PDAC and identifies a target whose inhibition generates impressive synergistic responses in combination with immunochemotherapy. Given the poor response of PDAC to currently employed treatment regimens in the oncology clinic, the preclinical data from this study is likely to have tremendous translational potential in the event that acceptable levels of side-effect toxicity are reported in those treated with such enzyme inhibitors. This study also raises certain questions that could pave the way for future preclinical research efforts that could be centered on understanding (i) the importance of Pin1 in regulating the heterogeneity of CAFs within the TME, (ii) whether PDL1 induction upon Pin1 inhibition might represent one mechanism of adaptive resistance to immunotherapy, and (iii) whether the treatment regimens described in this study can also be extended to other carcinoma types that are largely refractory to immunochemotherapy.

\section{REFERENCES}

1. Sharma, P. \& Allison, J. P. Science 348, 56-61 (2015).

2. Brahmer, J. R. Clin. Adv. Hematol. Oncol. 10, 674-675 (2012).

3. Özdemir, B. C. et al. Cancer Cell 28, 831-833 (2015).

4. Rhim, A. D. et al. Cancer Cell 25, 735-747 (2014).

5. Koikawa, K. et al. Cell 184, 4753-4771.e27 (2021).

6. Lu, Z. \& Hunter, T. Cell Res. 24, 1033-1049 (2014).

7. Zhou, X. Z. \& Lu, K. P. Nat. Rev. Cancer 16, $463-478$ (2016).

8. Batlle, E. \& Massague, J. Immunity 50, 924-940 (2019).

9. Wang, H. et al. Nat. Chem. Biol. 15, 42-50 (2019).

\section{COMPETING INTERESTS}

R. A. W. has a consulting agreement with Verastem Inc together with holding shares of this company.

\section{ADDITIONAL INFORMATION}

Correspondence and requests for materials should be addressed to Robert A. Weinberg.

Reprints and permission information is available at http://www.nature.com/reprints 